\title{
Modification of C11, C28, C2,3,23 or C2,23,28 Functional Groups on Asiatic Acid and Evaluation of Hepatoprotective Effects
}

\author{
Long-Xuan Zhao ${ }^{\dagger}$ Hyeung-geun Park,,$\stackrel{+}{+}$ Sang-sup Jew,${ }^{\ddagger}$ Mi Kyeong Lee, ${ }^{\ddagger}$ Young Choong Kim,,${ }^{\ddagger}$ Pritam Thapa, \\ Radha Karki, Yurngdong Jahng, Byeong-Seon Jeong," and Eung-Seok Lee*

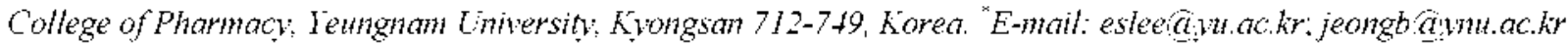 \\ ${ }^{\dagger}$ Department of Chemistry, Liaoning Kormal Liniversity, Dalian, Liaoning 116-029, P. R. China \\ ${ }^{\ddagger}$ College of Pharmacy, Seoul National Lniversitw, Seoul 151-742, Korea \\ Received April 9, 2007
}

\begin{abstract}
For the development of novel hepatoprotective agents. C1 1. C28. C2,3,23 or C2,23,28 functional groups on asiatic acid were modified. and their hepatoprotective effects were evaluated. Most of the prepared compounds displayed potent hepatoprotective activities against $\mathrm{CCl}_{4}$ - and galactosamine ( $\mathrm{GaNN}$ )-induced hepatotoxicity. Especially, compounds $\mathbf{1 6}$ and $\mathbf{2 0}$ showed the most significant hepatoprotective effects against GaIN-induced hepatotoxicity ( $54.2 \%$ and $46.4 \%$ protection at $50 \mathrm{mM}$. respectively).
\end{abstract}

Key Words : Asiatic acid, Hepatoprotective effect, Structural modification, Hepatotoxicity

\section{Introduction}

Liver is one of the most important organs in the human body performing excretion and metabolism. Acute and chronic hepatic disease has been recently increased but efficient drugs without side effects have not been developed. It has been reported that ursolic and oleanoic acid which have ursane structure showed strong hepatoprotective activity ${ }^{1-6}$ Asiatic acid. whose structure is derived from ursane skeleton. is one of the triterpenoids isolated from Centella Asiatica. ${ }^{7}$ and has moderate hepatoprotective activity. ${ }^{\S}$ Asiatic acid can be easily prepared from hydrolysis of asiaticoside. Modification of functional groups of asiatic acid may provide valuable information of structure-activity relationships for the development of novel hepatoprotective agents. Previously. we reported modifications of $\mathrm{C} 2$ and $\mathrm{C} 2,3,23.28$ functional group on asiatic acid and evaluation of their hepatoprotective effects. In connection with these studies. we modified C11. C28. C2.3.23 or C2.3.28 functional groups on asiatic acid and evaluated their hepatoprotective effects.

\section{Experimental}

Material and methods. All reagents were purchased from Aldrich Chemical (www. sigma-aldrich.com) and used without further purification. Unless otherwise indicated. anlydrous solvent were distilled over $\mathrm{CaH}_{2}$ or sodium benzophenone ketyl prior to use. Thin-layer chromatography (TLC) and column chromatography were performed with Kieselgel 60 $\mathrm{F}_{534}$ (Merck) and silica gel Kieselgel 60. (230-400 mesh. Merck), respectively. Compounds containing aromatic ring were visualized on TLC plates with UV light. and compounds containing oxygen were visualized on TLC plates with $p$ anisaldehyde staining solution. Nuclear magnetic resonance (NMR) spectra were taken on a Bruker AMX $250 \mathrm{MHz}$ for ${ }^{1} \mathrm{H}$ NMR and $62.5 \mathrm{MHz}$ for ${ }^{13} \mathrm{C}$ NMR. and tetrametlyy lsilane (TMS) was used as an intennal standard. Chemical shifts $(\delta)$ were recorded in ppm. and coupling constants $(J)$ in $\mathrm{Hz}$. Melting points were determined in open capillary tubes on electrothermal 1A 9100 digital melting point apparatus and were uncorrected.

2 $\alpha, 3 \beta, 23$-Triacetoxyurs-12-ene-28-oic acid (2). To a stirred solution of $1(3.00 \mathrm{~g} .6 .14 \mathrm{mmol})$ in pyridine $(60 \mathrm{~mL})$ was added acetic anlydride $(5.80 \mathrm{~mL}, 61.40 \mathrm{mmol})$. The mixture was stirred at $20^{\circ} \mathrm{C}$ for $8 \mathrm{~h}$. After dilution with ethyl acetate $(250 \mathrm{~mL})$. the mixture was washed with aqueous $1 \mathrm{M}$ $\mathrm{HCl}(50 \mathrm{~mL} \times 5)$ and saturated $\mathrm{CuSO}_{4}(25 \mathrm{~mL} \times 2)$ and saturated $\mathrm{NaCl}$ solution $(50 \mathrm{~mL})$. The organic phase was dried over anhydrous magnesium sulfate. Filtration and evaporation of solvent at reduced pressure gave light yellow solid. which

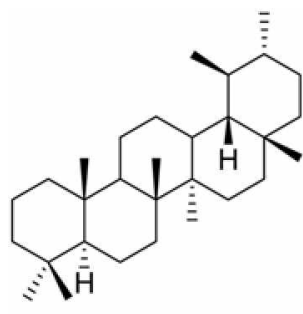

Ursane

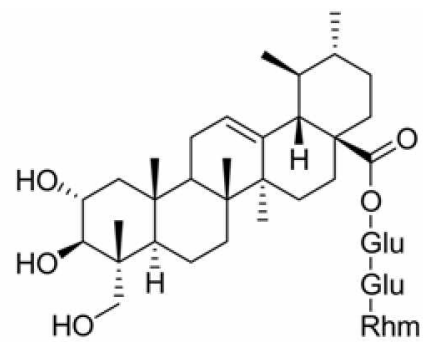

Asiaticoside

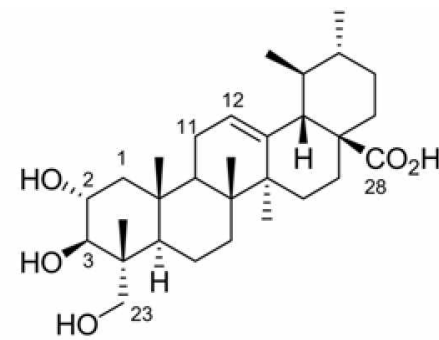

Asiatic acid (1)

Figure 1. Structures of ursane, asiaticoside and asiatic acid. 
was purified by silica gel chrontatography with a gradient elution of EtOAc/n-hexane (1:3. viv) to yield a white solid (2.2 g. 58.3\%).

TLC (EtOAc:n-hexane $=1: 2, v \mathrm{v}), \mathrm{R}_{f}=0.25 .{ }^{1} \mathrm{H}$ NMR $\left(250 \mathrm{MHz}, \mathrm{CDCl}_{3}\right) \delta 5.24$ (br. $\left.\mathrm{lH}\right), 5.15-5.09(\mathrm{~m}, \mathrm{lH}), 5.08$ (d. $J=10.3 \mathrm{~Hz}, \mathrm{IH}) .3 .82($ d. $J=11.7 \mathrm{~Hz}, \mathrm{IH}) .3 .58(\mathrm{~d}, J=$ $11.7 \mathrm{~Hz} . \mathrm{lH}) .2 .19(\mathrm{~d}, J=11.1 \mathrm{~Hz} .1 \mathrm{H}), 2.09,2.03 .1 .98$. $1.10,1.07,0.88,0.76$ (s. each $3 \mathrm{H}) .0 .94$ (d. $J=5.6 \mathrm{~Hz} .3 \mathrm{H}$ ). 0.85 (d. $J=6.4 \mathrm{~Hz} .3 \mathrm{H}) .{ }^{13} \mathrm{C} \mathrm{NMR}\left(62.5 \mathrm{MHz}, \mathrm{CDCl}_{3} . \mathrm{Cl}\right.$. C13. C28 carbon only) $\delta 178.65 .138 .34,125.67$.

$2 \alpha, 3 \beta, 23$-Triacetoxyurs-11-oxo-12-ene-28-0ic acid (3). A solution of $2(1.73$ g. $2.81 \mathrm{mmol})$ and $\mathrm{Na}_{2} \mathrm{Cr}_{2} \mathrm{O}_{7} \cdot 2 \mathrm{H}_{2} \mathrm{O}$ $(2.10 \mathrm{~g} .7 .04 \mathrm{mmol})$ in $100 \mathrm{~mL}$ of acetic acid was refluxed for $5 \mathrm{~h}$. The mixture was cooled to $20^{\circ} \mathrm{C}$ and neutralized

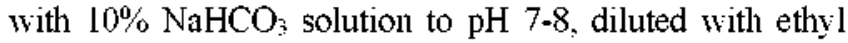
acetate $(150 \mathrm{~mL})$ and washed with water $(50 \mathrm{~mL} \times 5)$. and saturated $\mathrm{NaCl}$ solution $(50 \mathrm{~mL})$. The organic phase was dried over andhydrous magnesium sulfate. Filtration and evaporation of solvent at reduced pressure gave light yellow solid. which was purified by silica gel chromatography with a gradient elution of $\mathrm{CH}_{2} \mathrm{Cl}_{2} / \mathrm{MeOH}(30: \mathrm{l}$. v.v) to yield a light yellow solid $(1.64$ g. $92.7 \%)$.

$\mathrm{TLC}\left(\mathrm{CH}_{2} \mathrm{Cl}_{2}: \mathrm{MeOH}=9: \mathrm{L} . \mathrm{v} \cdot \mathrm{v}\right), \mathrm{R}_{f}=0.55 .{ }^{1} \mathrm{H}$ NMR $(250$ $\left.\mathrm{MHz} . \mathrm{CDCl}_{3}\right) \delta 5.62$ (s. $\mathrm{lH}$ ) 5.29 (dt. $J=11,4.5 \mathrm{~Hz} . \mathrm{lH}$ ). $5.05(\mathrm{~d} . J=10.3 \mathrm{~Hz}, 1 \mathrm{H}) .3 .82(\mathrm{~d}, J=11.9 \mathrm{~Hz}, \mathrm{lH}) .3 .61(\mathrm{~d}$. $J=11.9 \mathrm{~Hz}, \mathrm{lH}$ ). 3.2 (dd. $J=12.8 .4 .6 \mathrm{~Hz} .1 \mathrm{H}), 2.09,2.02$. $1.96,1.30 .1 .29 .0 .90,0.89(\mathrm{~s}$, each $3 \mathrm{H}) .0 .97(\mathrm{~d}, J=6.0 \mathrm{~Hz}$. $3 \mathrm{H}), 0.86(\mathrm{~d} . J=6.4 \mathrm{~Hz} .3 \mathrm{H}) .{ }^{13} \mathrm{C}$ NMR $(62.5 \mathrm{MHz}$. pyridine- $d_{s} \mathrm{C} 11 . \mathrm{Cl} 2, \mathrm{C} 13 . \mathrm{C} 28$ carbon only) $\delta 199.67$. 179.23. 164.54, 131.34

$2 \alpha_{3} 3 \beta, 23$-Trihydroxyurs-11-oxo-12-ene-28-oic acid (4). To a stirred solution of $3(0.93 \mathrm{~g}, 1.48 \mathrm{~mm}$ ol $)$ in methanol $(100 \mathrm{~mL})$ and water $(20 \mathrm{~mL})$ was added potassium carbonate (1.23 g. $8.88 \mathrm{mmol}$ ) and stirred for $6 \mathrm{~h}$ at $20^{\circ} \mathrm{C}$. The nixture was neutralized with aqueous $1 \mathrm{M} \mathrm{HCl}$ to $\mathrm{pH} 2-3$. The solvent was evaporated under reduced pressure to give a light yellow solid, which was purified by silica gel chromatography with a gradient elution of $\mathrm{CH}_{2} \mathrm{Cl}_{2} / \mathrm{MeOH}$ (9:1. v.v) to yield a white solid $(0.60$ g. $80.6 \%)$.

$\mathrm{TLC}\left(\mathrm{CH}_{2} \mathrm{Cl}_{2}: \mathrm{MeOH}=9: 1 . \mathrm{vv}\right), \mathrm{R}_{f}=0.21 .{ }^{1} \mathrm{H}$ NMR $(250$ MHz. pyridine- $\left.d_{s}\right) \delta 6.0(\mathrm{~s}, 3 \mathrm{H}), 4.38(\mathrm{~m} . \mathrm{lH}) .4 .25$ (d. $J=$ $9.7 \mathrm{~Hz}, 2 \mathrm{H}$ ). 3.73 (dd. $J=8.5 .4 .0 \mathrm{~Hz}, 1 \mathrm{H}$ ). 3.7 (d. $J=10.5$ Hz, 1H). 2.75 (s. lH). 2.66 (d. $J=11.3 \mathrm{~Hz}, 1 \mathrm{H}), 1.46,1.28$. 1.22. 1.06 .0 .85 (s. each $3 \mathrm{H}), 0.80(\mathrm{~d} J=6.4 \mathrm{~Hz}, 3 \mathrm{H}) .{ }^{13} \mathrm{C}$ NMR $\left(62.5 \mathrm{MHz}\right.$, pyridine- $d_{5}, \mathrm{Cl1} . \mathrm{Cl} 2, \mathrm{Cl} 3 . \mathrm{C} 28$ carbon only) $\delta 199.81,179.58 .164 .24 .131 .07$.

$2 \alpha$-Benzyloxy-3/,23-isopropylidenedioxy-28-hydroxyurs-12-ene (6). To the solution of $5(1.88 \mathrm{~g} .2 .97 \mathrm{mmol})^{9}$ in dry tetralydrofuran $(20 \mathrm{~mL})$ was added lithium aluminum hydride $(1.0 \mathrm{M}$ solution in tetrahydrofuran. $3.0 \mathrm{~mL}$ ) and refluxed for $1 \mathrm{~h}$. The mixture was filtered and evaporated under reduced pressure to remove THF. The residue was diluted with ethyl acetate $(100 \mathrm{~mL})$ and washed with water $(60 \mathrm{~mL} \times 3)$ and saturated $\mathrm{NaCl}$ solution $(50 \mathrm{~mL})$. The organic phase was dried over anhyydrous magnesium sulfate. Filtration and evaporation of solvent at reduced pressure gave a light yellow solid. which was purified by silica gel chromatograply with a gradient elution of EtOAc/n-hexane (1:4.v.v) to yield a white solid ( $1.5 \mathrm{~g} .83 .5 \%$ ).

TLC (EtOAc: 7 -hexane $=1: 5 . \mathrm{vv}) . \mathrm{R}_{f}=0.15 .{ }^{\mathrm{l}} \mathrm{H}$ NMR (250 MHz. $\left.\mathrm{CDCl}_{3}\right) \delta 7.35-7.25$ (m. $\left.5 \mathrm{H}\right) .5 .14$ (br, $\left.\mathrm{lH}\right), 4.82$. $4.59(\mathrm{AB}$ quartet, $J=11.7 \mathrm{~Hz} .2 \mathrm{H}), 3.64-3.46(\mathrm{~m}, 5 \mathrm{H}), 3.19$ (d. $J=10.9 \mathrm{~Hz} .1 \mathrm{H}$ ). 2.09 (d. $1 \mathrm{H}) .1 .48 .1 .47$ (s, each $3 \mathrm{H}$ ). 1.10. 1.10. 1.03. 0.96. 0.93 (s, each 3H). ${ }^{13} \mathrm{C}$ NMR $(62.5$ $\mathrm{MHz}$, pyridine- $d_{5} . \mathrm{C} 12, \mathrm{Cl} 3 . \mathrm{C} 28$ carbon only) $\delta 138.87$, $124.31,69.73$

$2 \alpha$-Benzyloxy-3 $\beta, 23,28$-trihydroxyurs-12-ene (7). To the solution of $6(1.30 \mathrm{~g} .2 .15 \mathrm{mmol})$ in THF $(50 \mathrm{~mL})$ was added aqueous $1 \mathrm{M} \mathrm{HCl}(8 \mathrm{~mL})$ at $20^{\circ} \mathrm{C}$ and stirred for $10 \mathrm{~h}$. The mixture was evaporated under reduced pressure to remove THF. The residue was purified by silica gel chromatograply with a gradient elution of $\mathrm{EtOAc} / n$-hexane $(1: 2 . v: \mathrm{v})$ to yield a white solid ( $1.1 \mathrm{~g} .90 .6 \%$ ).

TLC $($ EtOAc:n-hexane $=1: 2 . \mathrm{vv}) . \mathrm{R}_{f}=0.15 .{ }^{1} \mathrm{H}$ NMR ( $250 \mathrm{MHz} . \mathrm{CDCl}_{3}$ ) $\delta 7.38-7.28$ (m. $5 \mathrm{H}$ ). 5.15 (br. $\mathrm{lH}$ ). 4.68 , 4.45 (AB quartet, $J=11.2 \mathrm{~Hz}, 2 \mathrm{H}), 3.64(\mathrm{~d}, J=10.5 \mathrm{~Hz}$, IH). $3.59-3.50$ (m. $3 \mathrm{H}) .3 .40(\mathrm{~d}, J=10.6 \mathrm{~Hz} . \mathrm{lH}) .3 .19$ (d. $J$ $=11.2 \mathrm{~Hz} .1 \mathrm{H}) .2 .18(\mathrm{~d} .1 \mathrm{H}), 1.10,1.05 .0 .99 .0 .93,0.89(\mathrm{~s}$, each $3 \mathrm{H}) .{ }^{13} \mathrm{C}$ NMR $\left(62.5 \mathrm{MHz}\right.$, pyridine- $d_{5} . \mathrm{Cl} 2 . \mathrm{Cl} 3, \mathrm{C} 28$ carbon only) $\delta 139.68,123.81 .69 .55$.

$\mathbf{2} \alpha, 3 \beta, 23,28$-Tetrahydroxyurs-12-ene (8). $7(0.87 \mathrm{~g} .1 .54$ mmol) was dissolved in absolute $\mathrm{MeOH}(20 \mathrm{~mL})$, and $10 \%$ $\mathrm{Pd} / \mathrm{C}$ (100 mg) was added to the solution under argon atmosphere. The mixture was hydrogenolyzed for $3 \mathrm{~h}$ under $60 \mathrm{psi}$. The mixture was filtered using Celite pad to remove $\mathrm{Pd} / \mathrm{C}$ and evaporated under reduced pressure. The residue was purified by silica gel cluromatography with a gradient elution of $\mathrm{CH}_{2} \mathrm{Cl} / \mathrm{MeOH}(20: \mathrm{l}$. v.v) to yield a white solid $(0.65$ g. $88.9 \%)$.

TLC $\left(\mathrm{CH}_{2} \mathrm{Cl}_{2}: \mathrm{MeOH}: \mathrm{AcOH}=9: 1: 0.1\right.$, v.v. $), \mathrm{R}_{f}=0.41$. ${ }^{1} \mathrm{H}$ NMR (250 MHz. pyridine- $d_{5}$ ) $\delta 5.19$ (br, lH). 4.3-4.15 $(\mathrm{m}, 3 \mathrm{H}) .3 .89$ (d. $J=10.5 \mathrm{~Hz}, \mathrm{lH}), 3.72(\mathrm{~d} . J=10.5 \mathrm{~Hz} . \mathrm{lH})$. $3.59(\mathrm{~s}, 1 \mathrm{H}), 3.45(\mathrm{~d}, J=10.5 \mathrm{~Hz}, 1 \mathrm{H}), 1.12,1.10,1.08,1.03$, 0.91 (s. each $3 \mathrm{H}), 0.90(\mathrm{~d}, J=6.4 \mathrm{~Hz} .3 \mathrm{H}) .{ }^{13} \mathrm{C}$ NMR $(62.5$ $\mathrm{MHz}$, pyrridine-ds. $\mathrm{Cl} 2, \mathrm{Cl} 3 . \mathrm{C} 28$ carbon only) $\delta 138.99$, $124.28,69.87$.

3ק,23-Diacetoxyurs-2-oxo-12-ene-28-oic acid (10). To a stirred solution of $9(150 \mathrm{mg} .0 .31 \mathrm{mmol})^{9}$ in THF $(5 \mathrm{~mL})$ was added 4-dimethylaminopyridine ( $10 \mathrm{mg}$ ). After stirring for $30 \mathrm{~min}$ at $20^{\circ} \mathrm{C}$, acetic anlyydride $(0.17 \mathrm{~mL}, 1.85 \mathrm{mmol})$ was added. and the mixture was stirred for $2 \mathrm{~h}$ at $20^{\circ} \mathrm{C}$. The mixture was evaporated under reduced pressure to remove THF. The residue was diluted with ethyl acetate $(50 \mathrm{~mL})$ and washed with water $(20 \mathrm{~mL} \times 2)$ and saturated $\mathrm{NaCl}$ solution $(20$ $\mathrm{mL}$ ). The organic phase was dried over anhydrous magnesium sulfate. Filtration and evaporation of solvent at reduced pressure gave a light yellow solid. which was purified by silica gel chromatography with a gradient elution of EtOAc/ $n$-hexane $(1: 2 . v \mathrm{y})$ to yield a white solid $(130 \mathrm{mg} .73 .5 \%)$

TLC $($ EtOAc $n$-hexane $=1: 2 . \mathrm{vv}) . \mathrm{R}_{f}=0.22 .{ }^{1} \mathrm{H}$ NMR $\left(250 \mathrm{MHz} . \mathrm{CDCl}_{3}\right) \delta 5.25$ (br. $\mathrm{lH}$ ), $5.23(\mathrm{~s}, 1 \mathrm{H}) .4 .05$ (d. $J=$ $11.7 \mathrm{~Hz}, 1 \mathrm{H}), 3.74(\mathrm{~d}, J=11.8 \mathrm{~Hz}, 1 \mathrm{H}) .2 .47(\mathrm{~d}, J=12.3$ Hz. 1H). 2.17. 2.11. 1.12. 0.96. 0.80. 0.77 (s. each $3 \mathrm{H}$ ). 0.93 $(\mathrm{d} . J=6.0 \mathrm{~Hz}, 3 \mathrm{H}), 0.86(\mathrm{~d} . J=6.3 \mathrm{~Hz}, 3 \mathrm{H}) .{ }^{13} \mathrm{C}$ NMR $(62.5$ 
$\mathrm{MHz} . \mathrm{CDCl}_{3}, \mathrm{C} 2 . \mathrm{C} 3, \mathrm{C} 12 . \mathrm{C} 13 . \mathrm{C} 23, \mathrm{C} 28$ carbon only) $\delta$ 204.41. 183.84, 171.01. 170.62, 138.55, 125.32.

$3 \beta$-Hydroxy-23-acetoxyurs-2-0x0-12-ene-28-0ic acid (11). To a stirred solution of $9(200 \mathrm{mg} .0 .41 \mathrm{mmnol})$ in THF (10 $\mathrm{mL})$ was added acetyl chloride $(0.088 \mathrm{~mL} .1 .23 \mathrm{mmnol})$ at $0^{\circ} \mathrm{C}$. After stirring for $5 \mathrm{~min}$, triethylamine $(0.3+\mathrm{mL} .2 .46$ mmol) was added at $0^{\circ} \mathrm{C}$, and the mixture was stirred for $12 \mathrm{~h}$ at $20^{\circ} \mathrm{C}$. The nixture was evaporated under reduced pressure to remove THF. The residue was diluted with ethyl acetate $(50 \mathrm{~mL})$ and washed with water $(20 \mathrm{~mL} \times 2)$ and saturated $\mathrm{NaCl}$ solution $(20 \mathrm{~mL})$. The organic phase was dried over anhydrous magnesium sulfate. Filtration and evaporation of solvent at reduced pressure gave a light yellow solid. which was purified by silica gel chromatography with a gradient elution of $\mathrm{EtOAc} / n$-hexane $(1: 1, \mathrm{vv})$ to yield a white solid (90 mig. $41.5 \%$ ).

TLC (EtOAc;n-hexane $=1: 1, \mathrm{vv}), \mathrm{R}_{f}=0.21 .{ }^{1} \mathrm{H}$ NMR $\left(250 \mathrm{MHz}, \mathrm{CDCl}_{3}\right) \delta 5.25(\mathrm{br}, 1 \mathrm{H}), 4.27(\mathrm{~s} .1 \mathrm{H}) .4 .17(\mathrm{~d} . J=$ $11.5 \mathrm{~Hz} . \mathrm{lH}) .3 .86(\mathrm{~d}, J=11.5 \mathrm{~Hz}, \mathrm{lH}) .2 .53(\mathrm{~d} . J=12.3 \mathrm{~Hz}$. lH), 2.11. 1.12. 0.96, 0.80, $0.77(\mathrm{~s}$, each $3 \mathrm{H}), 0.93(\mathrm{~d}, J=6.0$ $\mathrm{Hz}, 3 \mathrm{H}) .0 .86(\mathrm{~d}, J=6.3 \mathrm{~Hz} .3 \mathrm{H}),{ }^{13} \mathrm{C}$ NMR $(62.5 \mathrm{MHz}$. $\mathrm{CDCl}_{3}, \mathrm{C} 2, \mathrm{C} 12 . \mathrm{Cl} 3, \mathrm{C} 23, \mathrm{C} 28$ carbon only) $\delta 211.31$. 183.75. 171.01, 138.63, 125.26.

38,23-Diacetoxyurs-12-ene-28-oic acid (13). To a stirred solution of $12(150 \mathrm{mg}, 0.32 \mathrm{mmol})^{9}$ in THF $(5 \mathrm{~mL})$ was added 4-dimethylaminopyridine (10 mg). After stirring for $30 \mathrm{~min}$. acetic anhydride $(0.3 \mathrm{~mL}, 3.17 \mathrm{mmol})$ was added. and the mixture was stirred for $2 \mathrm{~h}$ at $20^{\circ} \mathrm{C}$. The mixture was evaporated under reduced pressure to remove THF. The residue was diluted with ethyl acetate $(50 \mathrm{~mL})$ and washed with water $(20 \mathrm{~mL} \times 2)$ and saturated $\mathrm{NaCl}$ solution $(20 \mathrm{~mL})$. The organic phase was dried over anhydrous magnesium sulfate. Filtration and evaporation of solvent at reduced pressure gave a light yellow solid. which was purified by silica gel chromatography with a gradient elution of EtOAC/ $n$-hexane (l:4. v: $\mathrm{v})$ to yield a white solid ( $140 \mathrm{mg} .78 .6 \%$ ).

TLC (EtOAc: $n$-hexane $=1: 2, \mathrm{viv}) . \mathrm{R}_{f}=0.35 .{ }^{1} \mathrm{H}$ NMR $(250$ $\left.\mathrm{MHz}, \mathrm{CDCl}_{3}\right) \delta 5.24$ (br. $\left.\mathrm{lH}\right) .4 .79(\mathrm{~m}, \mathrm{lH}), 3.88(\mathrm{~d} . J=11.6$ Hz. $1 \mathrm{H}) .3 .69$ (d. $J=11.6 \mathrm{~Hz}, 1 \mathrm{H}) .2 .19(\mathrm{~d} . J=11.2 \mathrm{~Hz}, 1 \mathrm{H}$ ). $2.07,2.03,1.07 .0 .99,0.82 .0 .77$ (s. each $3 \mathrm{H}), 0.94$ (d. $J=$ $6.5 \mathrm{~Hz} .3 \mathrm{H}), 0.86($ d. $J=6.3 \mathrm{~Hz} .3 \mathrm{H}) .{ }^{13} \mathrm{C}$ NMR $(62.5 \mathrm{MHz}$. $\mathrm{CDCl}_{3}, \mathrm{C} 3, \mathrm{C} 12, \mathrm{C} 13 . \mathrm{C} 23 . \mathrm{C} 28$ carbon only) $\delta 183.97$. 171.44. 171.12, 138.35, 126.02

$3 \beta$ Hydroxy-23-trimethylacetoxyurs-12-ene-28-0ic acid (14). To a stirred solution of 12 ( $150 \mathrm{mg} .0 .32 \mathrm{nmmol}$ ) in THF $(5 \mathrm{~mL})$ was added 4-dimethylaminopyridine $(10 \mathrm{mg})$. After stirring for $30 \mathrm{~min}$, trimethylacetic andydride $(0.64 \mathrm{~mL} .3 .17$ mmol) was added. and the mixture was stirred for $2 \mathrm{~h}$ at $20^{\circ} \mathrm{C}$. The mixture was evaporated under reduced pressure to remove THF. The residue was diluted with ethyl acetate $(50 \mathrm{~mL})$ and washed with water $(20 \mathrm{~mL} \times 2)$ and saturated $\mathrm{NaCl}$ solution $(20 \mathrm{~mL})$. The organic phase was dried over anlydrous magnesium sulfate. Filtration and evaporation of solvent at reduced pressure gave a light yellow solid. which was purified by silica gel chromatography with a gradient elution of EtOAc/n-hexane $(1: 2 . \mathrm{v} \mathrm{v})$ to yield a white solid (120 mg. 67.3\%).
TLC $($ EtOAc: $n$-hexane $=1: 2 . \mathrm{vv}) . \mathrm{R}_{f}=0.20 .{ }^{1} \mathrm{H}$ NMR (250 MHz, $\left.\mathrm{CDCl}_{3}\right) \delta 5.25$ (br. $\mathrm{lH}$ ) 4.18 (d. $J=11.5 \mathrm{~Hz}$, IH). $3.81($ d. $J=11.4 \mathrm{~Hz}, \mathrm{lH}) .3 .39(\mathrm{~m} . \mathrm{lH}) .2 .19$ (d. $J=$ $11.1 \mathrm{~Hz}, \mathrm{lH}), 1.23$ (s. $3 \mathrm{H}), 1.06,0.97,0.78,0.76$ (s, each $3 \mathrm{H}) .0 .94(\mathrm{~d}, J=7.2 \mathrm{~Hz}, 3 \mathrm{H}), 0.86($ d. $J=6.4 \mathrm{~Hz} .3 \mathrm{H}) .{ }^{13} \mathrm{C}$ NMR (62.5 MHz. $\mathrm{CDCl}_{3} . \mathrm{Cl} 2 . \mathrm{Cl} 3, \mathrm{C} 23, \mathrm{C} 28$ carbon only) $\delta 183.78 .179 .16,138.26,126.19$.

Methyl $2 \alpha, 23$-diacetoxy-3 $\beta$-hydroxyurs-12-ene-28-oate (16). To a stirred solution of $15(0.50 \mathrm{~g} .1 .00 \mathrm{mmol})^{\vartheta}$ and acetyl chloride $(1.06 \mathrm{~mL}, 14.90 \mathrm{mmol})$ in THF $(15 \mathrm{~mL})$ was added triethylamine $(1.5 \mathrm{~mL}$. $14.90 \mathrm{mmol})$ at $0^{\circ} \mathrm{C}$. The mixture was stirred for $3 \mathrm{~h}$ at $20^{\circ} \mathrm{C}$. The mixture was evaporated under reduced pressure to remove THF. The residue was diluted with ethyl acetate $(60 \mathrm{~mL})$ and washed with water $(30 \mathrm{~mL} \times$ 3) and saturated $\mathrm{NaCl}$ solution $(30 \mathrm{~mL})$. The organic phase was dried over anlydrous magnesium sulfate. Filtration and evaporation of solvent at reduced pressure gave a light yellow solid, which was purified by silica gel chromatography with a gradient elution of $\mathrm{EtOAc} / n$-hexane $(1: 2, v \mathrm{v})$ to yield a white solid ( $230 \mathrm{mg} .39 .2 \%$ ).

TLC (EtOAc: $n$-hexane $=1: 2 . \mathrm{vv}) . \mathrm{R}_{f}=0.21 .{ }^{1} \mathrm{H}$ NMR $\left(250 \mathrm{MHz}, \mathrm{CDCl}_{3}\right) \delta 5.26(\mathrm{t}, J=3.4 \mathrm{~Hz}, \mathrm{lH}), 5.02(\mathrm{~m}, \mathrm{lH})$, $4.08(\mathrm{~d}, J=11.5 \mathrm{~Hz} . \mathrm{lH}) .3 .94(\mathrm{~d}, J=11.4 \mathrm{~Hz}, \mathrm{lH}) .3 .61(\mathrm{~s}$, $3 \mathrm{H}) .3 .50(\mathrm{~d} . J=10.1 \mathrm{~Hz} . \mathrm{lH}), 2.23(\mathrm{~d}, J=11.3 \mathrm{~Hz} . \mathrm{lH})$, 2.08. $2.07(\mathrm{~s}$, each $3 \mathrm{H}) .1 .07 .1 .07,0.85 .0 .75$ (s, each $3 \mathrm{H})$. $0.94(\mathrm{~d} . J=5 \mathrm{~Hz} .3 \mathrm{H}) .0 .84(\mathrm{~d} . J=6.2 \mathrm{~Hz} .3 \mathrm{H}) .{ }^{13} \mathrm{C}$ NMR (62.5 MHz, CDCl $, \mathrm{C} 2, \mathrm{C} 12 . \mathrm{Cl} 3, \mathrm{C} 23 . \mathrm{C} 28$ carbon only) $\delta$ $178.40,171.86 .171 .40 .138 .68,125.12$.

Methyl $3 \beta, 23$-isopropylidenedioxyurs-2-oxo-12-ene-28oate (18). A solution of pyridinium dichromate $(56 \mathrm{mg})$ and acetic anhydride $(0.05 \mathrm{~mL})$ in dry $\mathrm{CH}_{2} \mathrm{Cl}_{2}(10 \mathrm{~mL})$ was stirred $30 \mathrm{~min}$ at $20^{\circ} \mathrm{C}$. To the reaction solution was added 17 (100 mg, $0.18 \mathrm{mmol})^{8}$ in dry $\mathrm{CH}_{2} \mathrm{Cl}_{2}(5 \mathrm{~mL})$ slowly and refluxed for $2 \mathrm{~h}$. The mixture was diluted with ethyl acetate $(80 \mathrm{~mL})$ and filtered to remove precipitate and washed with water $(30 \mathrm{~mL} \times 3)$ and saturated $\mathrm{NaCl}$ solution $(30 \mathrm{~mL})$. The organic phase was dried over anhy drous magnesium sulfate. Filtration and evaporation of solvent at reduced pressure gave a light yellow solid. which was purified by silica gel chromatograply with a gradient elution of $\mathrm{EtOAc} / n$-hexane $(1: 4 . \mathrm{vv})$ to yield a white solid $(71.8 \mathrm{mg} .73 .8 \%)$.

TLC $($ EtOAc: $n$-hexane $=1: 4, \mathrm{vv}), \mathrm{R}_{f}=0.22 .{ }^{1} \mathrm{H}$ NMR (250 MHz. $\left.\mathrm{CDCl}_{3}\right) \delta 5.25$ (br, lH). 4.40 (s. lH), 3.60 (s, $3 \mathrm{H}$ ). $3.70,3.59$ (AB quartet. $J=10.4 \mathrm{~Hz} .2 \mathrm{H}$ ). $2.25(\mathrm{~d}, J=$ $10.8 \mathrm{~Hz}, 1 \mathrm{H}$ ). 2.39 .2 .13 (AB quartet. $J=12.4 \mathrm{~Hz}, 2 \mathrm{H}$ ). 0.95 $(\mathrm{d} . J=5.6 \mathrm{~Hz}, 3 \mathrm{H}), 0.86(\mathrm{~d}, J=6.0 \mathrm{~Hz}, 3 \mathrm{H}), 1.52,1.45,1.14$. 1.05. 1.01. 0.74 (s. each $3 \mathrm{H}) .{ }^{13} \mathrm{C}$ NMR $\left(62.5 \mathrm{MHz}, \mathrm{CDCl}_{3}\right.$, C2. $\mathrm{C} 12, \mathrm{Cl} 3, \mathrm{C} 28$ carbon only) $\delta 211.59 .179 .11 .138 .87$. 125.08

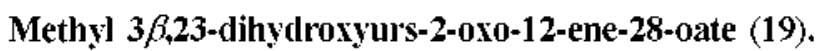
To the solution of $18(30 \mathrm{mg} .0 .055 \mathrm{mmol})$ in THF $(0.16$ $\mathrm{mL})$ was added aqueous $1 \mathrm{M} \mathrm{HCl}(5 \mathrm{~mL})$ and stirred for $2 \mathrm{~h}$ at $20^{\circ} \mathrm{C}$. The mixture was evaporated under reduced pressure to remove THF. The residue was diluted with ethyl acetate $(20 \mathrm{~mL})$. washed with water $(5 \mathrm{~mL} \times 2)$ and saturated $\mathrm{NaCl}$ solution $(10 \mathrm{~mL})$. The organic phase was dried over andydrous magnesium sulfate. Filtration and evaporation of solvent at 
reduced pressure gave a light yellow solid. which was purified by silica gel chromatography with a gradient elution of EtOACl $n$-hexane $(1: 2, v i)$ to yield a white solid $(27.8 \mathrm{mg}, 89.3 \%)$.

TLC (EtOAc: 7 -hexane $=1: 2, v \mathrm{v}), \mathrm{R}_{f}=0.20 .{ }^{1} \mathrm{H}$ NMR $\left(250 \mathrm{MHz}, \mathrm{CDCl}_{3}\right) \delta 5.25$ (br. $1 \mathrm{H}$ ) 4.36 (s, lH). $3.6 \mathrm{l}$ (s. $3 \mathrm{H}) .3 .52(\mathrm{~s}, 2 \mathrm{H}) .2 .50(\mathrm{~d}, J=12.2 \mathrm{~Hz}, \mathrm{lH}), 2.25(\mathrm{~d} . J=11.2$ $\mathrm{Hz}, 1 \mathrm{H}), 2.12($ d. $J=12.3 \mathrm{~Hz} . \mathrm{lH}) .1 .13 .0 .92,0.75,0.60(\mathrm{~s}$. each $3 \mathrm{H}) .0 .95(\mathrm{~d}, J=5.6 \mathrm{~Hz}, 3 \mathrm{H}), 0.86(\mathrm{~d} . J=6.4 \mathrm{~Hz} .3 \mathrm{H})$. ${ }^{13} \mathrm{C}$ NMR $\left(62.5 \mathrm{MHz}, \mathrm{CDCl}_{3} . \mathrm{C} 2 . \mathrm{Cl} 2 . \mathrm{Cl} 3, \mathrm{C} 28\right.$ carbon only) $\delta 211.65 .177 .98 .138 .66,125.01$.

Methyl $3 \beta$-hydroxy-23-acetoxyurs-2-oxo-12-ene-28-0ate (20). To a stirred solution of 19 (300 $\mathrm{mg} .0 .60 \mathrm{mmol})$ in $\mathrm{CH}_{2} \mathrm{Cl}_{2}(5 \mathrm{~mL})$ was added acetyl chloride $(0.128 \mathrm{~mL}, 1.80$ mmol) at $0^{\circ} \mathrm{C}$. After stirring for $5 \mathrm{~min}$. triethylamine $(0.5 \mathrm{~mL}$. $3.60 \mathrm{mmol}$ ) was added at $0^{\circ} \mathrm{C}$, and the mixture was stirred for $2 \mathrm{~h}$ at $20^{\circ} \mathrm{C}$. The mixture was evaporated under reduced pressure to remove THF. The residue was diluted with ethyl acetate $(50 \mathrm{~mL})$ and washed with water $(20 \mathrm{~mL} \times 2)$ and saturated $\mathrm{NaCl}$ solution $(30 \mathrm{~mL})$, the organic phase was dried over anlydrous magnesium sulfate. Filtration and evaporation of solvent at reduced pressure gave a light yellow solid. which was purified by silica gel chrontatography with a gradient elution of EtOAc/n-hexane (1:4. viv) to yield a white solid (158 mg. $48.5 \%$ ).

TLC (EtOAc: $n$-hexane $=1: 4, v v), R_{f}=0.21 .{ }^{1} \mathrm{H}$ NMR $\left(250 \mathrm{MHz} . \mathrm{CDCl}_{3}\right) \delta 5.26(\mathrm{t} . J=3.4 \mathrm{~Hz} . \mathrm{lH}), 4.26(\mathrm{~s}, \mathrm{lH})$. $4.18($ d. $J=11.5 \mathrm{~Hz} . \mathrm{lH}) .3 .86(\mathrm{~d}, J=11.5 \mathrm{~Hz} . \mathrm{IH}) .3 .60(\mathrm{~s}$. $3 \mathrm{H}), 2.53(\mathrm{~d}, J=12.3 \mathrm{~Hz}, \mathrm{lH}) .2 .26(\mathrm{~d} . J=11.2 \mathrm{~Hz}, \mathrm{lH})$. $2.14(\mathrm{~d}, J=12.3 \mathrm{~Hz}, 1 \mathrm{H}) .2 .11$ (s. $3 \mathrm{H}), 1.13,0.92 .0 .75,0.66$ (s. each $3 \mathrm{H}) .0 .95(\mathrm{~d}, J=5.6 \mathrm{~Hz}, 3 \mathrm{H}), 0.86(\mathrm{~d}, J=6.4 \mathrm{~Hz}$. $3 \mathrm{H}),{ }^{13} \mathrm{C}$ NMR $(62.5 \mathrm{MHz}, \mathrm{CDCl}, \mathrm{C} 2 . \mathrm{Cl} 2, \mathrm{C} 13 . \mathrm{C} 23 . \mathrm{C} 28$ carbon only) $\delta 211.28,178.31,170.97 .138 .90 .125 .03$.

Methyl $3 \beta$-hydroxy-23-propionyloxyurs-2-oxo-12-ene28-0ate (21). To a stirred solution of 19 ( $200 \mathrm{mg}, 0.40 \mathrm{mmol}$ ) in $\mathrm{CH}_{2} \mathrm{Cl}_{2}(5 \mathrm{~mL})$ was added propionyl chloride $(0.104 \mathrm{~mL}$. $1.20 \mathrm{mmol}$ ) at $0^{\circ} \mathrm{C}$. After stirring for $5 \mathrm{~min}$. trietlyylamine $\left(0.33 \mathrm{~mL} .2 .40 \mathrm{mmol}\right.$ ) was added at $0^{\circ} \mathrm{C}$. and the nixture was stirred for $2 \mathrm{~h}$ at $20^{\circ} \mathrm{C}$. The mixture was evaporated under reduced pressure to remove $\mathrm{CH}_{2} \mathrm{Cl}_{3}$. The residue was diluted with ethyl acetate $(50 \mathrm{~mL})$ and washed with water $(20 \mathrm{~mL} \times$ 2) and saturated $\mathrm{NaCl}$ solution $(30 \mathrm{~mL}$ ). The organic phase was dried over anhyydrous magnesium sulfate. Filtration and evaporation of solvent at reduced pressure gave a light yellow solid. which was purified by silica gel chromatography with a gradient elution of EtOAc/n-hexane $(1: 4, \mathrm{v} v)$ to yield a white solid ( $54.5 \mathrm{mg} .22 .3 \%$ ).

TLC $($ EtOAc: $n$-hexane $=1: 4, \mathrm{v} v), \mathrm{R}_{f}=0.20 .{ }^{1} \mathrm{H}$ NMR $\left(250 \mathrm{MHz}, \mathrm{CDCl}_{3}\right) \delta 5.26$ (t. $\left.J=3.4 \mathrm{~Hz}, 1 \mathrm{H}\right), 4.27(\mathrm{~s}, 1 \mathrm{H})$. 4.21 (d. $J=11.5 \mathrm{~Hz} . \mathrm{lH}) .3 .86(\mathrm{~d}, J=11.5 \mathrm{~Hz} . \mathrm{lH}) .3 .61$ (s. $3 \mathrm{H}) .2 .53(\mathrm{~d} . J=12.3 \mathrm{~Hz} .1 \mathrm{H}), 2.40(\mathrm{q} . J=7.6 .2 \mathrm{H}) .2 .26(\mathrm{~d}$. $J=10.9 \mathrm{~Hz}, 1 \mathrm{H}) .2 .13($ d. $J=12.7 \mathrm{~Hz}, 1 \mathrm{H}) .1 .19$ (t. $J=7.5$ $\mathrm{Hz}, 3 \mathrm{H}), 1.12,0.92,0.75,0.67$ (s. each $3 \mathrm{H}), 0.95(\mathrm{~d}, J=5.6$ Hz. $3 \mathrm{H}) .0 .86(\mathrm{~d} . J=6.4 \mathrm{~Hz} .3 \mathrm{H}),{ }^{13} \mathrm{C}$ NMR $(62.5 \mathrm{MHz}$. $\mathrm{CDCl}_{3}, \mathrm{C} 2, \mathrm{C} 12 . \mathrm{Cl} 3, \mathrm{C} 23, \mathrm{C} 28$ carbon only) $\delta 211.31$. 178.32. $174.23,138.89,125.06$

Methyl $3 \beta$-hydroxy-23-trimethylacetoxyurs-2-0x0-12ene-28-oate (22). To a stirred solution of 19 (200 mg. 0.40 numol) in $\mathrm{CH}_{2} \mathrm{Cl}_{2}(5 \mathrm{~mL})$ was added pivaloyl chloride $(0.148$ $\mathrm{mL} .1 .20 \mathrm{mmol}$ ) at $0^{\circ} \mathrm{C}$. After stirning for $5 \mathrm{~min}$. trietlyylamine $(0.33 \mathrm{~mL}, 2.4 \mathrm{mmol})$ was added at $0^{\circ} \mathrm{C}$, and the mixture was stirred for $2 \mathrm{~h}$ at $20^{\circ} \mathrm{C}$. The mixture was evaporated under reduced pressure to remove $\mathrm{CH}_{2} \mathrm{Cl}_{2}$. The residue was diluted with ethyl acetate $(50 \mathrm{~mL})$ and washed with water $(20 \mathrm{~mL} \times$ 2) and saturated $\mathrm{NaCl}$ solution $(30 \mathrm{~mL})$. The organic phase was dried over anlyydrous magnesium sulfate. Filtration and evaporation of solvent at reduced pressure gave a light yellow solid. which was purified by silica gel chromatography with a gradient elution of EtOAc/n-hexane $(1: 5, \mathrm{vv})$ to yield a white solid (112.9 mg. 48.3\%)

TLC (EtOAc: 7 -hexane $=1.5 . \mathrm{vv}) . \mathrm{R}_{f}=0.21 .{ }^{\mathrm{H}} \mathrm{H}$ NMR $\left(250 \mathrm{MHz} . \mathrm{CDCl}_{3}\right) \delta 5.26(\mathrm{t}, J=3.4 \mathrm{~Hz} . \mathrm{lH}), 4.27(\mathrm{~s}, \mathrm{lH})$, $4.20(\mathrm{~d}, J=11.5 \mathrm{~Hz} . \mathrm{lH}) .3 .83(\mathrm{~d}, J=11.5 \mathrm{~Hz}, \mathrm{lH}) .3 .61(\mathrm{~s}$, $3 \mathrm{H}) .2 .54(\mathrm{~d} . J=12.2 \mathrm{~Hz} . \mathrm{lH}), 2.26(\mathrm{~d}, J=11.1 \mathrm{~Hz} . \mathrm{lH}$ ), 2.10 (d. $J=12.4 \mathrm{~Hz}, \mathrm{lH}$ ). 1.26 (s. $9 \mathrm{H}$ ). $1.10 .0 .92,0.75 .0 .67$ (s, each $3 \mathrm{H}) .0 .94$ (d. $J=6.0 \mathrm{~Hz}, 3 \mathrm{H}) .0 .86($ d. $J=6.4 \mathrm{~Hz}$, $3 \mathrm{H}) .{ }^{13} \mathrm{C}$ NMR $\left(62.5 \mathrm{MHz} . \mathrm{CDCl}_{3}, \mathrm{C} 2, \mathrm{C} 12 . \mathrm{Cl} 3 . \mathrm{C} 23 . \mathrm{C} 28\right.$ carbon only) $\delta 211.30,178.33 .178 .08,138.84,125.09$.

Primary cell cultured rat hepatocytes assay. Rat hepatocytes were prepared from male Wistar rats by a collagenase perfusion teclunique of Berry and Friend with minor modification. ${ }^{1 j} 24 \mathrm{~h}$ after the isolated rat hepatocytes were plated. the cultured cells were exposed to a culture containing $5 \mathrm{mM}$ of chloroform or glucosamine either with. or without the prepared compounds along with asiatic acid and silymarin. After $1.5 \mathrm{~h}$, the activities of glutamic pyruvic transaminase (GPT) released into the culture medium were determined by the method of Reitman-Frankel. ${ }^{11}$ All data are expressed as the mean $\pm \mathrm{SD}$. The evaluation of statistical significance was determined by "the one-ivay ANOVA" using a computerized statistical package. The data were considered to be statistically significant if the probability had a value of 0.05 or less.

\section{Results and Discussion}

Chemistry. For the preparation of the asiatic acid derivative whose methylene group at $\mathrm{C} .11$ position was modified to keto group allylic oxidation was performed Because olefinic carbon at C.12 position is known to be very resistant to epoxidation. catalytic hydrogenation. and dihydroxylation, we investigated to modify this position by allylic oxidation. since structurally similar compounds has been reported to be modified by allylic oxidation. ${ }^{\text {"2 }}$ Acetylation of asiatic acid (1) with acetic anthydride gave protected intermediate 2 in $58.3 \%$ yield. which was converted to $\alpha \beta$-unsaturated ketone 3 in $92.7 \%$ yield by the oxidation with $\mathrm{Na}_{2} \mathrm{Cr}_{2} \mathrm{O}_{7}$ in the presence of acetic acid. ${ }^{13}$ The final product, trilydrosy compound + was obtained in $80.6 \%$ yield by deacetylation of 3 (Scheme 1 ).

For the modification of C28-carboxylic acid moiety at $\mathrm{C} 28$ position (Scheme 2), intermediate $5^{8}$ was reduced with lithium aluninum lydride $\left(\mathrm{LiAlH}_{4}\right)$ to give hydroxymethyl intermediate 6 in $83.5 \%$ yield. Deprotection of acetonide group of 6 with aqueous $1 \mathrm{M} \mathrm{HCl}$ gave diol intermediate 7 in $90.6 \%$ yield. followed by deprotection of benẓ̇l moiety by hydrogenolysis 


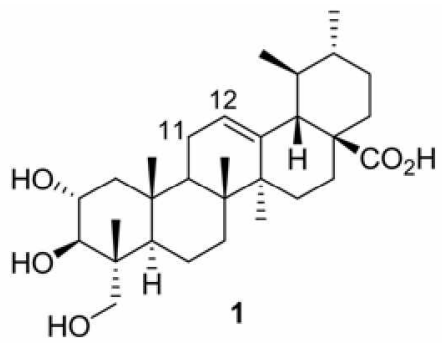

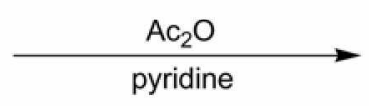

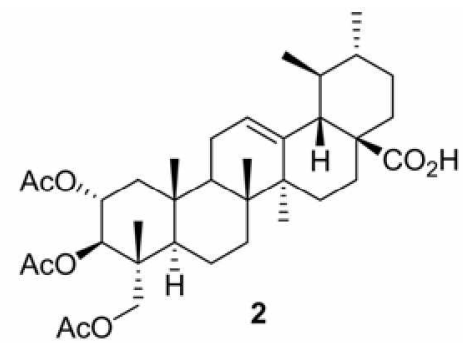

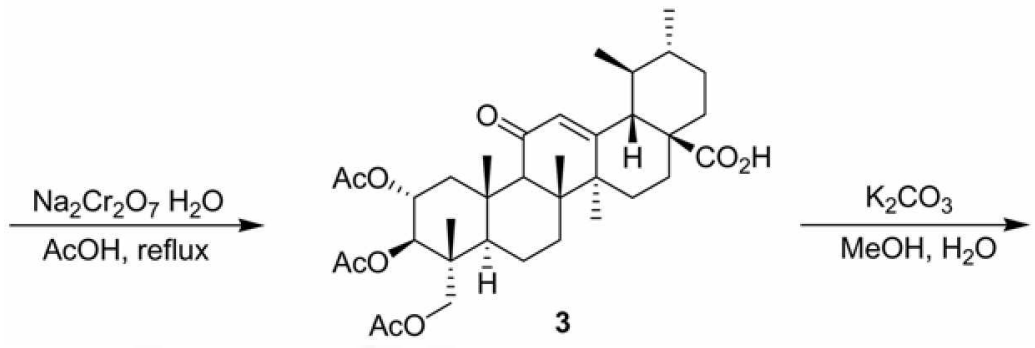

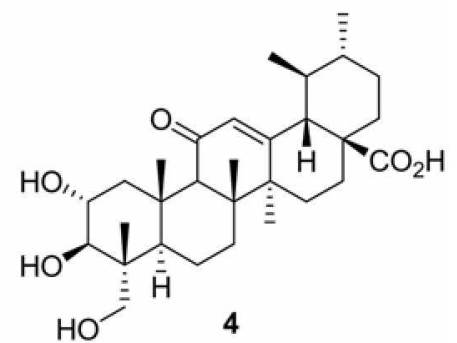

Scheme 1. Synthesis of C11-keto asiatic acid.

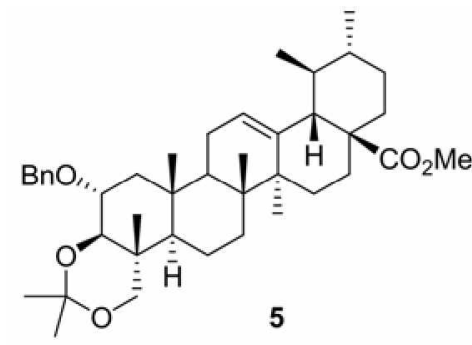

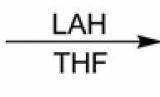

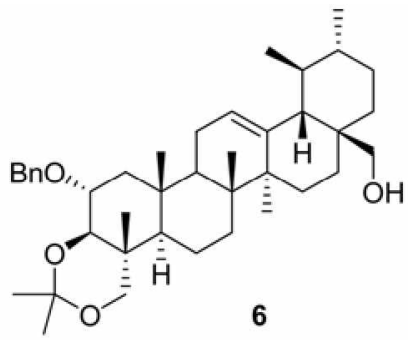

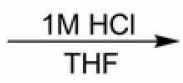

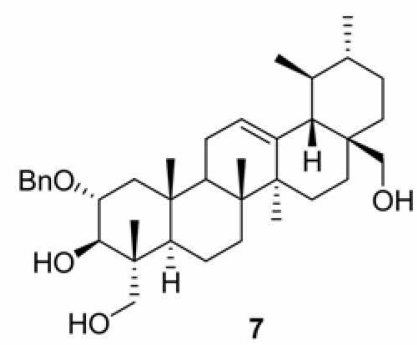<smiles>CCCCCCC(C)C</smiles>

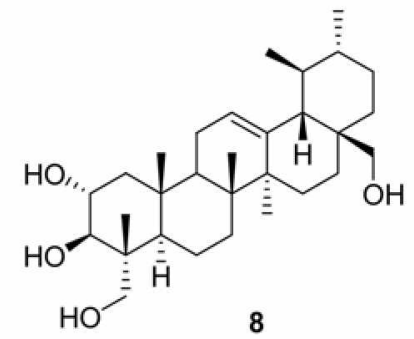

Scheme 2. Synthesis of C28-hydroxymethyl asiatic acid.

with $\mathrm{H}_{2}$ and $10 \% \mathrm{Pd} / \mathrm{C}$ gave a funal product 8 in $88.9 \%$ yield. in which carboxylic acid moiety on asiatic acid was converted to hyddroxymethyl moiety.

For the preparation of $\mathrm{C} 2$-oxo asiatic acid derivatives (10. 11) diol compound $9^{8}$ was treated with acetyl chloride or

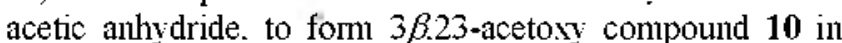
$73.5 \%$ yield or 23 -acetoxy compound 11 in $41.5 \%$ yield. respectively (Scheme 3).

For the preparation of C2-deoxygenated asiatic acid (13. 14). intermediate $12^{8}$ was treated with acetic anhydride or trimethylacetic anlhydride in the presence of DMAP, to afford $3 \beta .23$-diacetoxy substituted compound 13 in $78.6 \%$ yield and 23-trimethylacetoxy substituted conpound 14 in $67.3 \%$ yield respectively:

For the preparation of $2 \alpha 23$-dilydrosy substituted compound 16. methyl ester $15^{8}$ was treated with acetyl chloride and triethylamine in THF to provide 16 in $39.2 \%$ yield (Scheme 4).
For the preparation of 23-acyl substituted derivatives (2022). methyl ester $17^{8}$ was oxidized with $\mathrm{PDC}$ in $\mathrm{CH}_{2} \mathrm{Cl}_{2}$ under reflux to give $2-0$ xo compound 18 in $73.8 \%$ yield. Deprotection of 18 with aqueous $1 \mathrm{M} \mathrm{HCl}$ gave diol compound 19 in $89.3 \%$ yield. which was treated with acetyl chloride. propionyl chloride or pivaloyl chloride in $\mathrm{CH}_{2} \mathrm{Cl}_{2}$, to form 20. 21 or 22 in $48.5 \% .22 .3 \%$ and $48.3 \%$ y ield, respectively (Scheme 4).

Pharmacology. For the evaluation of hepatoprotective activities. hepatotoxicity was artificially induced by administration of galactosamine or $\mathrm{CCl}_{4}$ into primary cultured rat hepatocytes. Prepared compounds were administered with different concentration, and relative glutamic pynuvic transaminase (GPT) activities were observed according to recovery of enzyme activities by the Reitman-Frankel method." Hepatoprotective effect was indicated as \% value. (Nomal GPT activity: $100 \%$. intoxicated (JPT activity: $0 \%$ ). Hepato- 

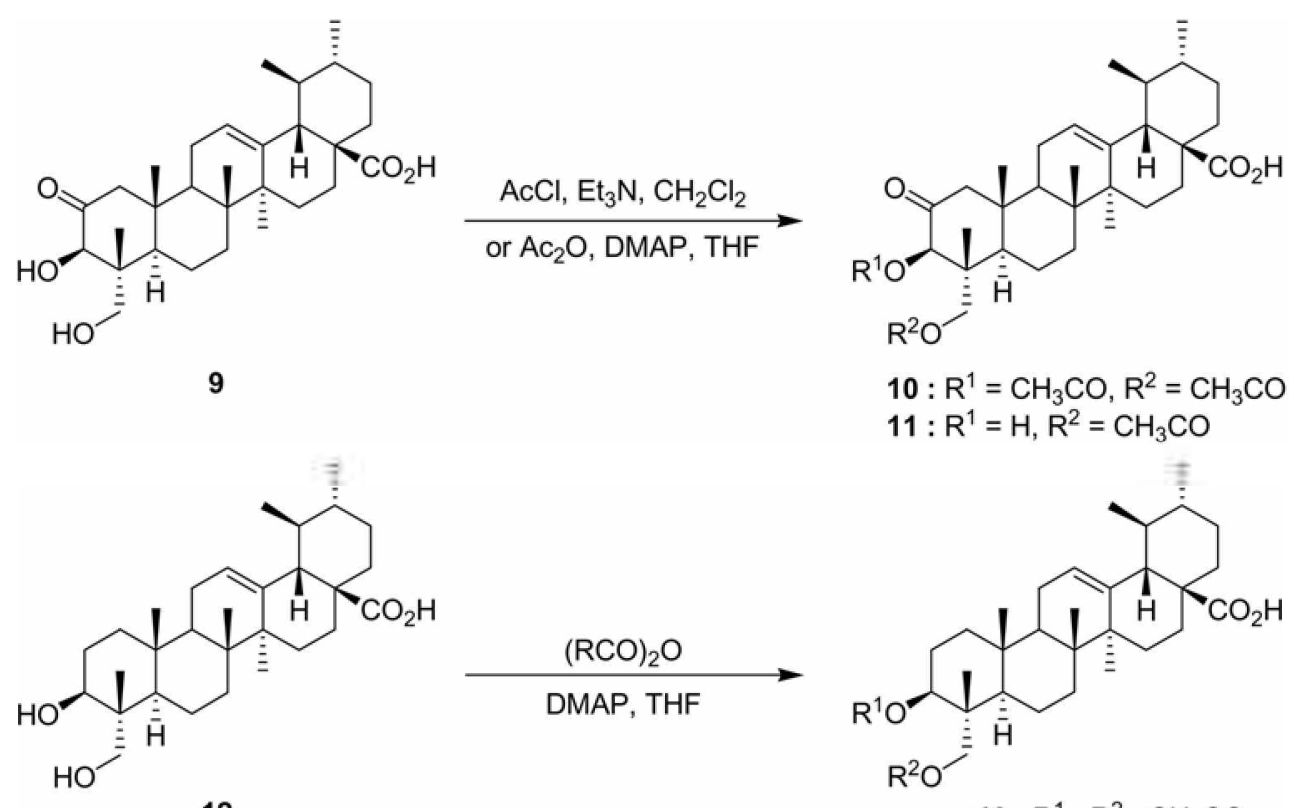

$10: \mathrm{R}^{1}=\mathrm{CH}_{3} \mathrm{CO}, \mathrm{R}^{2}=\mathrm{CH}_{3} \mathrm{CO}$

$11: \mathrm{R}^{1}=\mathrm{H}, \mathrm{R}^{2}=\mathrm{CH}_{3} \mathrm{CO}$

12

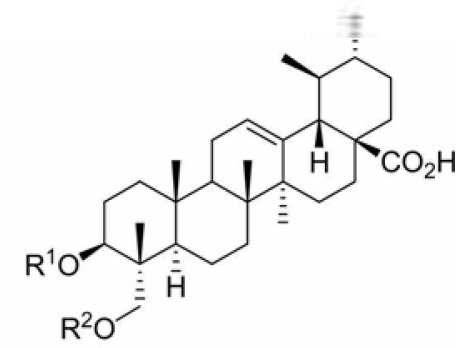

$13: \mathrm{R}^{1}=\mathrm{R}^{2}=\mathrm{CH}_{3} \mathrm{CO}$

$14 \cdot R^{1}=H_{1} R^{2}=t-84 C O$

Scheme 3. Synthesis of C2,3,23-modified asiatic acid.

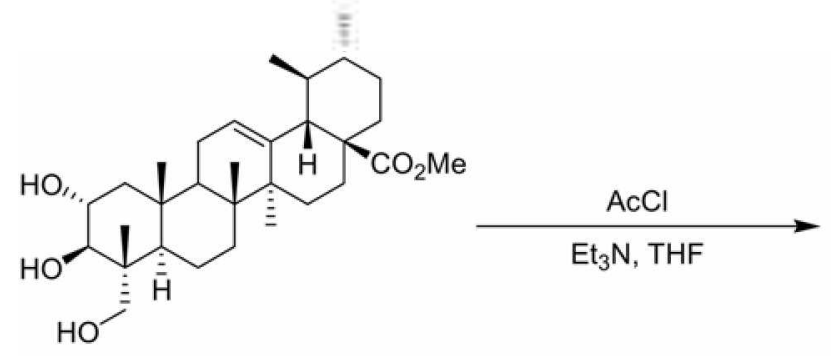

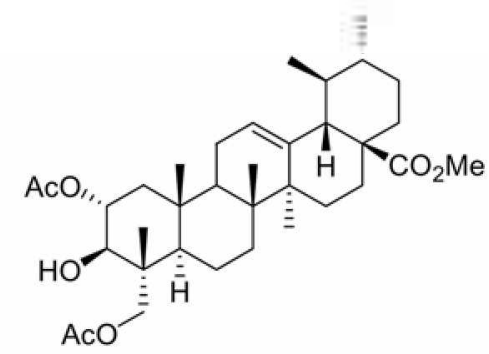

15

16

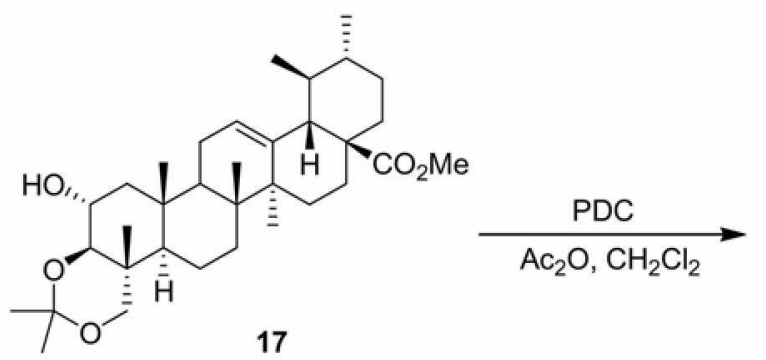

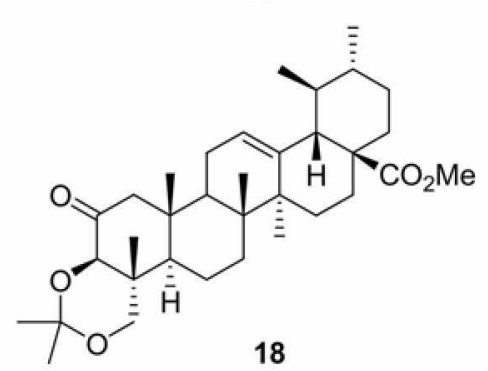

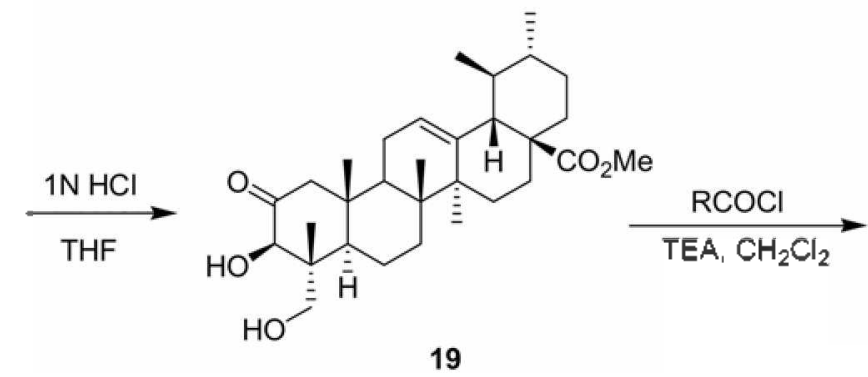

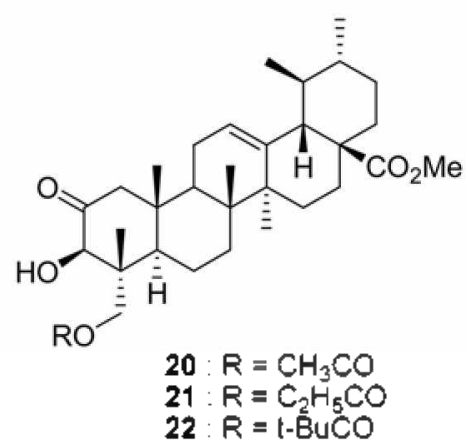

Scheme 4. Synthesis of $C 2,23,28$-modified asiatic acid

toxicity induced by $\mathrm{CCl}_{4}$ was reported to be due to lipid peroxide which was trichloromethyl free radical $\left(\mathrm{CCl}_{3}\right)$ metabolite bound with intracellular proteins and lipids by action of cytochrome P-450 dependent mixed oxidase. Hepatotoxicity 
Table 1. Hepatoprotective eflects of the prepared compounds

\begin{tabular}{ccc}
\hline \multirow{2}{*}{ Compound } & $\mathrm{CCl}_{4}$-induced & GalN-induced \\
\cline { 2 - 3 } & Protection $(\%)$ at $50 \mu \mathrm{M}$ Protection $(\%)$ at $50 \mu \mathrm{M}$ \\
\hline Asiatic acid $(\mathbf{1})$ & 1.0 & 23.1 \\
$\mathbf{3}$ & $\mathrm{NE}$ & 13.0 \\
$\mathbf{4}$ & 25.6 & $\mathrm{NE}$ \\
$\mathbf{8}$ & 39.7 & $\mathrm{NE}$ \\
$\mathbf{1 0}$ & 22.0 & 14.4 \\
$\mathbf{1 1}$ & 35.9 & 26.5 \\
$\mathbf{1 3}$ & $\mathrm{NE}$ & 22.3 \\
$\mathbf{1 4}$ & $\mathrm{NE}$ & 37.6 \\
$\mathbf{1 6}$ & $\mathrm{NE}$ & 54.2 \\
$\mathbf{2 0}$ & $\mathrm{NE}$ & 46.4 \\
$\mathbf{2 1}$ & $\mathrm{NE}$ & 21.0 \\
$\mathbf{2 2}$ & $\mathrm{NE}$ & 18.5 \\
Silvmarin & 54.7 & $\mathrm{NE}$ \\
\hline
\end{tabular}

NE: not effective.

induced by galactosamine has similarities with viral hepatitis in function and formation. Galactosamine was reported to inhibit RNA and protein synthesis, which was due to alteration of antount and metabolite of uracil nucleotides in liver. Galactosamine decreased the biosynthesis of biomacromolecules related to uracil nucleotides. such as UDP-glucuronic acid. which resulted in damage of related cells and cellular organelles.

Hepatoprotective activities of prepared compounds were evaluated. Silymarin was utilized as a reference compound to compare hepatoprotective activities with tested compounds. Silymarin has a very potent hepatoprotective activity (54.7\%) against $\mathrm{CCl}_{4}$-induced hepatotoxicity, but does not show any activity against galactosamine $(\mathrm{GaIN})$-induced hepatotoxicity. Asiatic acid nearly does not have hepatoprotective activity against $\mathrm{CCl}_{4}$-induced hepatoxicity. but moderate activity against $\mathrm{GaIN}$-induced hepatotoxicity. Most of the tested compounds showed considerable hepatoprotective activities (Table 1).

Modification of asiatic acid into keto group at $\mathrm{Cll}$ position (compound 4) and hyddroxylmethyl group at $\mathrm{C} 28$ position (compound 8) increased the hepatoprotective effect $(25.6 \%$ and $39.7 \%$, respectively) against $\mathrm{CCl}_{4}$-induced hepatotoxicity. Modification of hydroxyl group into keto group at $\mathrm{C} 2$ position (compounds 10.11) increased the hepatoprotective effect ( $22.0 \%$ and $35.9 \%$. respectively) against $\mathrm{CCl}_{4}$-induced hepatotoxicity and maintained hepatoprotective activity against GaINinduced hepatotoxicity. Acylation on $\mathrm{C} 23$ carbon (compounds 16. 20.21.22) or deoxygenation on $\mathrm{C} 2$ position (compounds 13. 14) of asiatic acid completely disappeared hepatoprotective effect against $\mathrm{CCl}_{4}$-induced hepatotoxicity. Generally. methyl ester derivatives on $\mathrm{C} 28$ position showed the sumilar or higher hepatoprotective activity against GaIN-induced hepatotoxicity. Especially, C2,C23-diacetyl methyl ester (compound 16) and C2-oxo.C23-acetyl methyl ester (compound 20) derivatives displayed the most potent hepatoprotective activity (54.2\% and $46.4 \%$, respectively) against GaIN-induced hepatotoxicity.

\section{Conclusions}

In conclusion. eleven asiatic acid derivatives by the modification of C11. C28. C2,3,23 or C2,23,28 functional groups were prepared, and their hepatoprotective activity against $\mathrm{CCl}_{4}$-induced and $\mathrm{GaNN}$-induced hepatotoxicity were evaluated. In addition, structure-activity relationship study of the modified compounds was performed. Among the prepared compounds. compound 16 and 20 showed the most significant hepatoprotective activity against GaIN-induced hepatotoxicity.

Acknowledgments. This work was supported by a grant (KRF-2006-005-J01101) from the Korea Research Foundation, R.O.K. The authors thank to Ministry of Education and Human Resources, Korea. for the support of BK21 scholarship of P. Thapa and R. Karki.

\section{References}

1. Liu. J.: Liu. Y. P.: Klaassen. C. D. J. Ethanopharntacol. 1994. 2 . 183.

2. Wang. B.: Jiang. Z. H. Chinese Phamaceutical Jownal 1992, 27. 393.

3. Yabuchi. T.: Tanaka. T.: Sasatsuka. T.: Yamahara, J.: Fujimura. H. IP 1987.62126149

4. Hikino. H: Ohsawa. I.: Kiso. Y: Oshima. Y. Planta Medica 1984.50 .353

5. Ma. X. H.: Zhao, Y. C.: Yin, L.: Han, D. W.: Wan. M. S. Acta Phamaceutica Sinica 1986. 21, 332

6. Shukla, B.: Viser, S.; Patnaik, G. K.: Tripathi. S. C.: Srimal. R. C.: Day. S.: Dobhal. P. C. Phwotherapy Res 1992.6.74.

7. Sastri. B. N. In The Healh of India, Raw Materials. C.S.I.R.: New Delhi. 1950: vol II. p 116.

8. Asiatic acid is also known as wound-healing agent. For the related references, see: (a) Beljanski. M.; Vapaille. N. Rev Eur: Enud. Clin. Biol. 1971, 16, 897. (b) Pointel, J. P, Boccalon. H.: Cloarec. M.: Ledevehat. J. M. Angrology 1987. 38. 46. (c) Bonte. F.: Dumas. M.: Chaudagne. C.: Meybeck. A. Am. Phorm. Fr: 1995. 53. 38. (d) Shim. P.-J.: Park. J.-H.: Chang. M.-S.: Lim. M.-J.: Kim. D.-H.: Jung. Y.-H; Jew, S.-S; Park, E.-H.; Kim. H. D. Bioorg. Med Chem. Lett 1996. 6. 2937. (e) Jeong. B.-S. Arch. Pham Res. 2006, 29. 556.

9. Jeong. B.-S.: Lee. M. K.: Kim. Y. C.: Lee. E.-S. Arch. Pham Res 2007. 30. 282 .

10. Berry. M. N.: Friend. D. S. J. Cell Biol. 1984. $\$ 3.506$

11. Reitman. S.; Frankel, S. A. Am. J. Chin. Pathol. 1957, 28.56.

12. Boiteau, P: Chanez. M. Dissentationes Pham 1963. 15. 189

13. Corey. E. J.: Ursprung. J. J. J. Am. Chem. Soc. 1956. 78. 183. 